\title{
Polarimetric Optical Fiber Sensors for Dynamic Strain Measurement in Composite Materials
}

\author{
A.W. Domański*, M. Bieda, P. Lesiak, P. Makowski, M. Szeląg, T. Poczęsny, \\ K. Prokopczuk, P. Sobotka, M. ChychŁowski, M. Sierakowski, T.R. Woliński \\ Faculty of Physics, Warsaw University of Technology, Koszykowa 75, 00-662 Warsaw, Poland

\begin{abstract}
Polarimetric optical fiber sensors possess possibility of temperature compensation, dynamic and integral system of strain monitoring, as well as low cost of photo-detecting elements. In the paper we present results of the sensor analysis with different kinds of birefringent optical fibers leading to an optimal setup for dynamic strain monitoring in composite materials. A great attention is put on parameters of the light sources like coherence and width of spectrum and their influence on dynamics of the strain sensor.
\end{abstract}

DOI: $10.12693 /$ APhysPolA.124.399

PACS: 42.79.Pw, 42.25.Bs, 42.81.Pa

\section{Introduction}

There are several types of fiber-optic sensors which may be used for strain sensing in composite materials [1-4]. The most popular are the sensors based on a fiber Bragg grating (FBG) [4-7]. The FBG measures strain at particular points and therefore even hundreds of FBGs may omit the crack place in a composite structure. It is important because mechanical properties of composites are specific and from macroscopic point of view they have homogeneous structure although combine various elements with different physical properties. The unique structure of the composite material enables possibility of breaking between different layers.

Polarimetric optical fiber sensors (POFS), which utilize highly birefringent (HB) fibers, belong to phase sensors in which polarization state of the output light depends on birefringence of the fiber. In our opinion HB fibers are more suitable for composite materials investigation than FBGs.

\section{Compensated optical fiber polarimetric sensor}

Due to depolarization of light in sensing fiber as well as temperature desensitization an additional compensating fiber should be added with crossed axes of birefringence. Linearly polarized light should be coupled into the sensing fiber with help of the polarization maintaining fiber. Such an all-fiber polarimetric sensor is shown in Fig. 1.

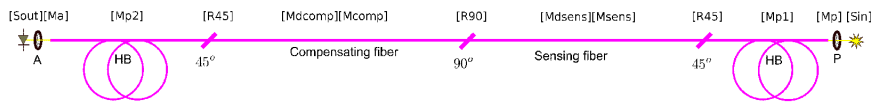

Fig. 1. All-fiber polarimetric sensor with compensated depolarization of light.

Modified Mueller-Stokes matrix equation including compensation and depolarization matrixes for all-fiber

*corresponding author; e-mail: domanski@if.pw.edu.pl polarimetric sensor is as follows:

$$
\begin{aligned}
& {\left[S_{\text {out }}\right]=\left[M_{\mathrm{A}}\right]\left[M_{\mathrm{p} 2}\right]\left[R_{45}\right]\left[M_{\mathrm{dcomp}}\right]\left[M_{\text {comp }}\right]\left[R_{90}\right]} \\
& \quad \times\left[M_{\text {dsens }}\right]\left[M_{\text {sens }}\right]\left[R_{45}\right]\left[M_{\mathrm{p} 1}\right]\left[M_{\mathrm{P}}\right]\left[S_{\text {in }}\right],
\end{aligned}
$$

where $\left[S_{\text {in }}\right]$ is a Stokes vector of the input light forming a different light source. $\left[M_{\mathrm{P}}\right]$ is a Mueller matrix of a linear polarizer to couple the light along one axis of the HB fiber. $\left[M_{\mathrm{p} 1}\right]$ is a Mueller matrix describing change of the Stokes vector due to propagation in a fiber medium. $\left[R_{45}\right]$ is a rotation matrix of the propagating wave into HB fiber to excite both axes. $\left[M_{\mathrm{dsens}}\right]$ and $\left[M_{\text {sens }}\right]$ are matrices describing depolarization and propagation in the sensing fiber, respectively. Next is rotation $\left[R_{90}\right]$ to change the axis of both propagating modes to orthogonal one. $\left[M_{\text {dcomp }}\right]$ and $\left[M_{\text {comp }}\right]$ are similar matrices to sensing with one exception, the influence on the fiber which they are describing is the same without a sensing factor, which is omitted in calculation for this reason. Last change of axis $\left[R_{45}\right]$ is to set propagating wave into the polarization maintaining fiber. $\left[M_{\mathrm{p} 2}\right]$ is matrix of the outgoing fiber. $\left[M_{\mathrm{A}}\right]$ is an analyzer matrix.

Modified Mueller-Stokes matrix for all-fiber polarimetric sensor is equal to

$$
\left[\begin{array}{l}
S_{0}^{\text {out }} \\
S_{1}^{\text {out }} \\
S_{2}^{\text {out }} \\
S_{3}^{\text {out }}
\end{array}\right]=\left[\begin{array}{cccc}
\frac{1}{2} & \frac{1}{2} & 0 & 0 \\
\frac{1}{2} & \frac{1}{2} & 0 & 0 \\
0 & 0 & 0 & 0 \\
0 & 0 & 0 & 0
\end{array}\right]
$$

$$
\begin{gathered}
\times\left[\begin{array}{cccc}
1 & 0 & 0 & 0 \\
0 & 1 & 0 & 0 \\
0 & 0 & \cos \left(\frac{2 \pi}{L_{\mathrm{B}}} L\right) & -\sin \left(\frac{2 \pi}{L_{\mathrm{B}}} L\right) \\
0 & 0 & \sin \left(\frac{2 \pi}{L_{\mathrm{B}}} L\right) & \cos \left(\frac{2 \pi}{L_{\mathrm{B}}} L\right)
\end{array}\right] \\
\times\left[\begin{array}{cccc}
1 & 0 & 0 & 0 \\
0 & 0 & -1 & 0 \\
0 & 1 & 0 & 0 \\
0 & 0 & 0 & 1
\end{array}\right]\left[\begin{array}{cccc}
1 & 0 & 0 & 0 \\
0 & P_{\mathrm{c}}^{*} & 0 & 0 \\
0 & 0 & P_{\mathrm{c}}^{*} & 0 \\
0 & 0 & 0 & P_{\mathrm{c}}^{*}
\end{array}\right]
\end{gathered}
$$




$$
\begin{aligned}
& \times\left[\begin{array}{cccc}
1 & 0 & 0 & 0 \\
0 & 1 & 0 & 0 \\
0 & 0 & \cos \left(\frac{2 \pi}{L_{\mathrm{B}}} L\right) & -\sin \left(\frac{2 \pi}{L_{\mathrm{B}}} L\right) \\
0 & 0 & \sin \left(\frac{2 \pi}{L_{\mathrm{B}}} L\right) & \cos \left(\frac{2 \pi}{L_{\mathrm{B}}} L\right)
\end{array}\right] \\
& \times\left[\begin{array}{cccc}
1 & 0 & 0 & 0 \\
0 & -1 & 0 & 0 \\
0 & 0 & -1 & 0 \\
0 & 0 & 0 & 1
\end{array}\right]\left[\begin{array}{llll}
1 & 0 & 0 & 0 \\
0 & P & 0 & 0 \\
0 & 0 & P & 0 \\
0 & 0 & 0 & P
\end{array}\right] \\
& \times\left[\begin{array}{cccc}
1 & 0 & 0 & 0 \\
0 & 1 & 0 & 0 \\
0 & 0 & \cos \left(\frac{2 \pi}{L_{\mathrm{B}}} L\right) & -\sin \left(\frac{2 \pi}{L_{\mathrm{B}}} L\right) \\
0 & 0 & \sin \left(\frac{2 \pi}{L_{\mathrm{B}}} L\right) & \cos \left(\frac{2 \pi}{L_{\mathrm{B}}} L\right)
\end{array}\right] \\
& \times\left[\begin{array}{cccc}
1 & 0 & 0 & 0 \\
0 & 0 & -1 & 0 \\
0 & 1 & 0 & 0 \\
0 & 0 & 0 & 1
\end{array}\right] \\
& \times\left[\begin{array}{cccc}
1 & 0 & 0 & 0 \\
0 & 1 & 0 & 0 \\
0 & 0 & \cos \left(\frac{2 \pi}{L_{\mathrm{B}}} L\right) & -\sin \left(\frac{2 \pi}{L_{\mathrm{B}}} L\right) \\
0 & 0 & \sin \left(\frac{2 \pi}{L_{\mathrm{B}}} L\right) & \cos \left(\frac{2 \pi}{L_{\mathrm{B}}} L\right)
\end{array}\right] \\
& \times\left[\begin{array}{cccc}
\frac{1}{2} & \frac{1}{2} & 0 & 0 \\
\frac{1}{2} & \frac{1}{2} & 0 & 0 \\
0 & 0 & 0 & 0 \\
0 & 0 & 0 & 0
\end{array}\right]\left[\begin{array}{c}
S_{0}^{\text {in }} \\
S_{1}^{\text {in }} \\
S_{2}^{\text {in }} \\
S_{3}^{\text {in }}
\end{array}\right],
\end{aligned}
$$

where $L$ is a length of the sensing and compensating fibers, $P=\exp \left(-\lambda L / L_{\mathrm{c}} L_{\mathrm{B}}\right)^{\alpha}$ and $P^{*}=$ $\exp \left(\lambda L / L_{\mathrm{c}} L_{\mathrm{B}}\right)^{\alpha} ; \alpha$ is equal to 1 or 2 dependent on Lorentzian (e.g. single mode LD) or Gaussian (e.g. LED, SLED) light sources; $L_{\mathrm{c}}$ means the coherence length of the light coupled to the $\mathrm{HB}$ fiber and $L_{\mathrm{B}}$ depends on strain $\varepsilon$ as follows [8]:

$$
L_{\mathrm{B}}(\varepsilon)=\frac{L_{\mathrm{B} 0}}{1+\frac{\varepsilon L_{\mathrm{B} 0}}{T_{\varepsilon} L}},
$$

in which $T_{\varepsilon}$ means a period of strain changing phase of output light of $2 \pi$.

In Eq. (2), instead of the matrix product $\left[M_{\text {dcomp }}\right]\left[M_{\text {comp }}\right]\left[R_{90}\right]\left[M_{\text {dsens }}\right]\left[M_{\text {sens }}\right]$ describing the sensing section followed by the compensating section, one could also use the following effective matrix [9]:

$$
\left[M^{11}(L)\right]=\left[\begin{array}{cccc}
1 & 0 & 0 & 0 \\
0 & 1 & 0 & 0 \\
0 & 0 & \operatorname{Re}(\gamma(L)) & -\operatorname{Im}(\gamma(L)) \\
0 & 0 & \operatorname{Im}(\gamma(L)) & \operatorname{Re}(\gamma(L))
\end{array}\right],
$$

which is a generalized version of the quasi-monochromatic matrix by Kortenski and Eftimov
[10] for highly birefringent single-mode optical fibers. Here, the length $L$ would mean the effective value affected by the birefringence changes induced in the sensing section. Unlike the matrix defined in [10], the form (4) is strictly correct for any light, as long as it is linearly polarized at the input plane, regardless of its bandwidth or the symmetry of its spectral profile. If the quasi-monochromatic approximation can be applied, the linear polarization at input is no longer required; the input Stokes vector, however, has to represent a pure polarization state (degree of polarization DOP $=1$ ). Within this approximation the complex degree of coherence of the source is expressed by

$$
\begin{aligned}
& \gamma(L)=\frac{\int_{0}^{\infty}|v(\omega)|^{2} \exp \left(-\mathrm{i}\left(\omega-\omega_{0}\right) \Delta \tau_{\mathrm{g}} L\right) \mathrm{d} \omega}{\int_{0}^{\infty}|v(\omega)|^{2} \mathrm{~d} \omega} \\
& \quad \times \exp \left(\mathrm{i} \Delta \beta\left(\omega_{0}\right) L\right),
\end{aligned}
$$

where $|v(\omega)|^{2}$ is the spectral intensity profile of the source with the central angular frequency $\omega_{0}$, $\Delta \tau_{\mathrm{g}}=\mathrm{d}(\Delta \beta) /\left.\mathrm{d} \omega\right|_{\omega_{0}}$ is the differential group delay of the birefringent fiber and $\Delta \beta=\beta_{y}-\beta_{x}=2 \pi / L_{\mathrm{B}}$ is the difference of propagation constants between the $\operatorname{LP}_{01}^{y}$ and $\mathrm{LP}_{01}^{x}$ polarization modes.

If the profile $|v(\omega)|^{2}$ can be regarded symmetrical with respect to $\omega_{0}$, we can approximate the matrix (4) by the form provided by Kortenski and Eftimov [10], which can be expressed by a product of the monochromatic Mueller matrix $[M]$ of the birefringent element for $\omega_{0}$ and a matrix of a nonuniform [11] depolarizer

$$
\begin{aligned}
& {\left[M^{0}(L)\right]} \\
& =\left[\begin{array}{cccc}
1 & 0 & 0 & 0 \\
0 & 1 & 0 & 0 \\
0 & 0 & |\gamma(L)| \cos \left(\frac{2 \pi}{L_{\mathrm{B}}} L\right) & -|\gamma(L)| \sin \left(\frac{2 \pi}{L_{\mathrm{B}}} L\right) \\
0 & 0 & |\gamma(L)| \sin \left(\frac{2 \pi}{L_{\mathrm{B}}} L\right) & |\gamma(L)| \cos \left(\frac{2 \pi}{L_{\mathrm{B}}} L\right)
\end{array}\right] \\
& =\left[\begin{array}{cccc}
1 & 0 & 0 & 0 \\
0 & 1 & 0 & 0 \\
0 & 0 & |\gamma(L)| & 0 \\
0 & 0 & 0 & |\gamma(L)|
\end{array}\right][M] .
\end{aligned}
$$

The formulae (6) are analogical to the product $\left[M_{\text {dsens }}\right]\left[M_{\text {sens }}\right]$ in $(2)$; apart from that $\left[M_{\text {dsens }}\right]$ describes a uniform depolarizer. It can be shown that both forms lead to the same DOP at the output plane, only the output state of polarization (SOP) differs. However, in the special case of the polarimetric sensor depicted in Fig. 1, where both polarization modes are equally excited in the sensing and the compensating sections, the second parameter of the Stokes vector of the passing light is $S_{1}=0$, therefore both forms become equivalent. Consequently, the expression (2) is sufficient to obtain correct form of the polarimetric signal.

\section{Dynamic strain characteristics of POFS}

Output signal from the compensated POFS (Fig. 1) may be calculated from the modified matrix Eq. (2): 


$$
\begin{aligned}
I_{0} & =\sqrt{\frac{\mu_{0}}{\varepsilon_{0}}} S_{0}^{\text {out }}=\sqrt{\frac{\mu_{0}}{\varepsilon_{0}}} \frac{1}{4}\left(S_{0}^{\text {in }}+S_{1}^{\text {in }}\right) \\
& \times\left[1+\exp \left(-\frac{\lambda \varepsilon}{L_{\mathrm{c}} T_{\varepsilon}}\right) \cos \left(\frac{2 \pi \varepsilon}{T_{\varepsilon}}\right)\right] .
\end{aligned}
$$

Measuring characteristics predicted for all-fiber polarimetric sensor in a composite material are shown in Fig. 2.

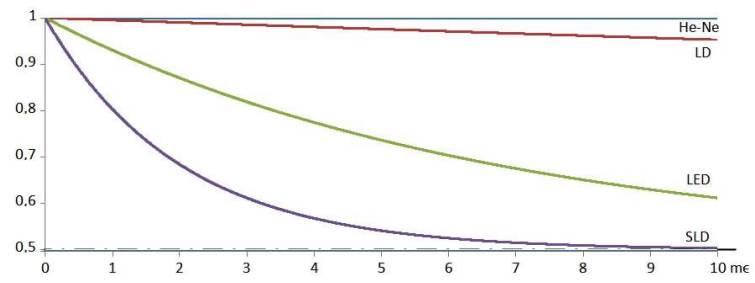

Fig. 2. Theoretical prediction of polarimetric fiber sensor dynamic (envelope) for different light sources: HeNe laser, $L_{\mathrm{c}}=2 \mathrm{~mm} ; \mathrm{LD}, L_{\mathrm{c}}=0.1 \mathrm{~mm} ;$ LED, $L_{\mathrm{c}}=10 \mu \mathrm{m} ; \mathrm{SLD}, L_{\mathrm{c}}=3 \mu \mathrm{m} ; L_{\mathrm{B}}=2 \mathrm{~mm}, L=0.5 \mathrm{~m}$.

Dynamics of these sensors may be expressed as follows:

$$
\eta=\frac{I_{0 \max }-I_{0 \min }}{I_{0 \max }+I_{0 \min }}
$$

and is equal to the DOP of the light outgoing from the sensor [12].

\section{Influence of the lamination process on dynamics of the fiber strain sensors embedded in composite materials}

In our previous paper [13] we proved that embedding of the polarimetric fiber sensor into the composite material can change both strain sensitivity and beat length of the fiber. These phenomena can influence sensor compensation since beat lengths of the sensing fiber and compensating fiber are not equal. Theoretical analysis for LED is presented in Fig. 3. Both sensing and compensating

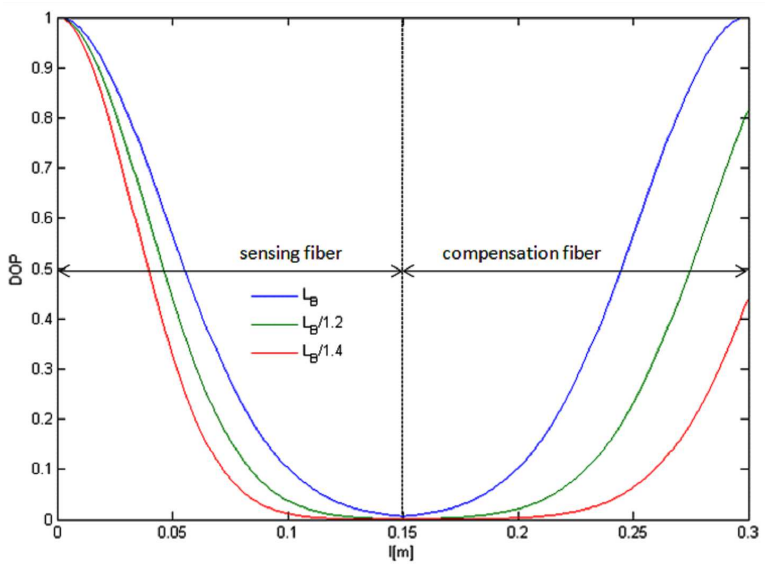

Fig. 3. Sensor compensation after lamination for different change in $L_{\mathrm{B}}$ after lamination. $\lambda=850 \mathrm{~nm}$, $L_{\mathrm{c}}=9.6 \mu \mathrm{m}, L_{\mathrm{B}}=4 \mathrm{~mm}$. fibers have the same lengths $L=0.15 \mathrm{~m}$. For a free-air sensor $L_{\mathrm{B}}$ does not change and full compensation is achieved. For the embedded sensors $L_{\mathrm{B}}$ is decreased and significant drop in output DOP is observed.

To achieve depolarization compensation in such a system, a compensation part of the sensor should be longer than its sensing part. However, this would cause the sensor to lose its temperature compensation. To prevent it, a light source with longer temporal coherence length must be used.

\section{Conclusions}

We have demonstrated that selected parameters of the polarimetric optical fiber sensors depend on length and birefringence of the sensing and compensating fibers as well as temporary coherence of light coupled into both fibers. The method of analysis of such sensors based on modified Mueller-Stokes matrix equation presented in the paper allows to predict main parameters of the sensor. It is a very important issue since the fiber embedded into composite materials changes its birefringence and therefore dynamics of the sensor may dramatically diminish.

\section{Acknowledgments}

This work is supported by the NCBiR grant no. PBS1/ B5/20/2012.

\section{References}

re1J.J. Guerin, M. Lequime, Proc. SPIE 2361, 224 (1994)

[2] G. Hegde, A. Asundi, Proc. SPIE 5852, 36 (2005).

[3] X. Chapeleau, H. Drissi-Habti, T. Tomiyama, Mat. Eval. 68, 409 (2010).

[4] A.W. Domanski, P. Lesiak, K. Milenko, D. Budaszewski, M. Chychlowski, S. Ertman, M. Tefelska, T.R. Wolinski, K. Jedrzejewski, L. Lewandowski, W. Jasiewicz, J. Helsztynski, A. Boczkowska, Acta Phys. Pol. A 116, 294 (2009).

[5] K. Schroeder, W. Ecke, J. Apitz, E. Lembke, G. Lenschow, Meas. Sci. Technol. 17, 1167 (2006).

[6] G. Wehrle, P. Nohama, H.J. Kalinowski, P.I. Torres, L.C.G. Valente, Meas. Sci. Technol. 12, 805 (2001).

[7] G. Hegde, A. Asundi, NDTEEE Int. 39, 320 (2006).

[8] W.J. Bock, A.W. Domański, T.R. Woliński, Appl. Opt. 29, 24 (1990).

[9] P.L. Makowski, A.W. Domański, Opt. Lett. 38, 1107 (2013).

[10] T. Kortenski, T. Eftimov, J. Mod. Opt. 37, 1413 (1990).

[11] R.A. Chipman, Proc. SPIE 5158, 184 (2003).

[12] A.W. Domanski, T.R. Wolinski, M.A. Karpierz, A. Kujawski, Proc. SPIE 2507, 204 (1995).

[13] P. Lesiak, G. Rajan, Y. Semenova, G. Farrell, A. Boczkowska, D. Budaszewski, M. Szeląg, A.W. Domański, T.R. Woliński, Proc. SPIE 7753, 4 (2011). 